\title{
SPECT Myocardial Perfusion Imaging: Poststress, End Systolic Images and the Ongoing Effort to Improve Diagnostic Accuracy
}

\author{
Henry Gewirtz
}

Division of Cardiology, Department of Medicine, Massachusetts General Hospital, Harvard Medical School, Boston, Massachusetts

I t is fair to state that interpretation of SPECT myocardial perfusion images, as with most scintigraphic studies, reflects a mixture of art and science. Increasingly, efforts have been, and are being, made to improve the diagnostic accuracy of SPECT myocardial perfusion imaging (MPI) by enhancing image quality while at the same time reducing radiation burden, via the scientific, technologic approach. Examples include the introduction of solid-state cadmium zinc telluride detectors, cardiac-optimized collimation, and improved image reconstruction software designed to mitigate factors such a patient motion, photon scatter, and tissue attenuation as well as spatial resolution and partial-volume effect $(1,2)$. Although these image-quality issues are much more pronounced in SPECT than in PET, PET is not immune. However, given the fundamental physics involved, PET imaging is better able to correct such errors, a fact previously noted by this group (3) and many others (4-6). It also has been appreciated for some time that apparent tracer distribution in single-photon myocardial perfusion

\section{See page 1882}

images, whether planar (7) or SPECT (8), may be altered by factors such a left ventricular volume and regional contraction independent of the true distribution of tracer in the myocardium $(7,8)$. Thus, increases in both left ventricular volume and regional contractile abnormality, especially apical akinesis or dyskinesis, have been shown to result in apparent perfusion defects despite the absence of true perfusion abnormality $(7,8)$. In this issue of The Journal of Nuclear Medicine, Kitkungvan et al. (9) have in essence turned the partial-volume effect to the scan interpreters' advantage by demonstrating a simple method for improving diagnostic accuracy of SPECT myocardial perfusion images with mild, borderline uptake defects. Such defects usually present the most problems in determining whether the image is abnormal. In contrast, accuracy in interpretation of more prominent defects was not improved by reference to the gated, end systolic image.

Received Jul. 30, 2015; revision accepted Jul. 31, 2015.

For correspondence contact: Henry Gewirtz, Cardiac Unit/Yawkey 5E, Massachusetts General Hospital, 55 Fruit St., Boston, MA 02114.

E-mail: gewirtz.henry@mgh.harvard.edu

Published online Aug. 27, 2015.

COPYRIGHT (C) 2015 by the Society of Nuclear Medicine and Molecular Imaging, Inc.

DOI: 10.2967/jnumed.115.164111

\section{WHAT TO LOOK FOR}

In reviewing the article by Kitkungvan et al., there are several issues to be aware of. The authors recognize certain limitations related to the retrospective nature of the work, including unidentified confounders and referral bias. They indicated they could not compensate for the latter but argued it did not materially affect the results of their study because the false-positive rate for wholecycle (i.e., ungated) images was comparable to that reported by others (10). They also were well aware of the limitations of using visually interpreted coronary angiograms ( $\geq 70 \%$ stenosis standard for abnormal) as the gold standard for the study but indicated they did so, again, to conform with real-world clinical practice. Thus, angiograms with visually moderate stenosis less than $70 \%$ would be classed as normal, a substantial problem of which the authors, who invented fractional flow reserve (11), are well aware but again apparently accepted for the purposes of the current paper to conform with standard clinical practice.

The authors indicated that both gated and ungated images were acquired in routine clinical fashion per American Society of Nuclear Cardiology guidelines. Thus, acquisition duration apparently was not increased beyond recommended duration to improve end systolic image quality. Further, no mention is made of how many cases had to be rejected for inadequate end systolic image quality due to arrhythmias such as atrial fibrillation or frequent ventricular ectopic activity. End systolic image quality also may be suboptimal in obese patients and others with prominent soft-tissue attenuation. So clearly, the method will not work for all patients but certainly is worth looking at because the data are gathered in any case.

As noted above and shown in Figure 4 of the article, the gated end systolic scan reduced the false-positive rate, thereby increasing the true-negatives, all as judged against the coronary angiogram, and so in turn enhanced diagnostic accuracy. The effect was confined to borderline defects and made no difference in interpretation of images with prominent perfusion abnormalities. The true-positive and false-negative rates were unchanged by use of the end systolic image, an observation largely preordained given the experimental design (scan vs. invasive coronary angiogram [ICA; gold standard]) and issue of referral bias for ICA.

Figure 1 of the article is of interest because it demonstrates how cardiac motion itself will contribute to the partial-volume effect and potentially create the impression of an inferior wall or apical defect - a point worth remembering because such motion is not captured by standard patient motion algorithms.

Likewise, Figure 2 is noteworthy because it displays what might be taken as an anterior wall defect in a mid-short-axis section (Fig. 2A) 
of the conventional image from a patient with normal coronary artery. However, the septal segment is clearly normal. Accordingly, given an intact native coronary circulation without coronary artery bypass graft surgery, the left anterior descending artery runs to the left ventricular apex in many cases, supplies the interventricular septum (anterior two thirds), and so is extremely unlikely to have a lesion ( $\geq 70 \%$ stenosis) in its proximal or even mid one third, which would limit flow to an isolated segment of anterior wall while sparing the septum and apex. Thus, one should be extremely circumspect in reading such apparent defects as true perfusion abnormalities. Artifact is far more likely and in the case shown with an opposite $180^{\circ}$ defect on the inferior wall (Fig. 2B), patient motion must be carefully considered.

\section{OPPORTUNITIES FOR IMPROVEMENT AND FUTURE DIRECTIONS}

Opportunities for improvements and future directions in MPI clearly rest in an area that the senior author of the present paper has been a leader in for more than $35 \mathrm{y}$ (12) and that even appears in the title of the current article, namely PET. Readers of the Journal are well aware of the widespread availability of the scanners and the relative ease of obtaining and using ${ }^{18} \mathrm{~F}$-FDG for clinical oncology as well as other indications. Although ${ }^{82} \mathrm{Rb}$ generators have made PET MPI feasible in many of the health care settings that lack on-site cyclotron capability, a variety of issues both with the ${ }^{82} \mathrm{Sr}$ generator and with the tracer itself have hampered widespread use. Administrative considerations related to cost, reimbursement, and organizational structure also have been, and in many cases continue to be, important barriers. In almost all centers with on-site cyclotron capability, ${ }^{13} \mathrm{~N}$-ammonia or ${ }^{15} \mathrm{O}$-water is used instead.

Hopefully, help is on the way. An ${ }^{18} \mathrm{~F}$-labeled tracer, flurpiridaz (13-15), is currently in phase 3 clinical trials. What is truly attractive about the tracer is its potential for use in making quantitative measurements of absolute myocardial blood flow $(13,15)$, which is the future of MPI, as reviewed in a recent state-of-the-art paper by the senior author of the current publication along with a substantial number of leaders in the field (16). Results of a phase 3 clinical trial were reported (invited presentation) at the International Conference on Nuclear Cardiology and Cardiac CT meeting in Madrid in May 2015 (17). This trial compared the diagnostic accuracy of PET ${ }^{18} \mathrm{~F}$-flurpiridaz with SPECT ${ }^{99 \mathrm{~m}} \mathrm{Tc}-$ methoxyisobutylisonitrile MPI for the detection of anatomically significant coronary artery disease (assessed by ICA). Quantitative measurements of absolute myocardial blood flow were not used nor was coronary stenosis severity assessed physiologically. Nonetheless, area-under-the-curve analysis of the diagnostic accuracy data demonstrated statistically significant, superior results with ${ }^{18}$ F-flurpiridaz versus SPECT ${ }^{99 m}$ Tc-methoxyisobutylisonitrile. It was also reported that the accuracy of ${ }^{18} \mathrm{~F}$-flurpiridaz was superior to that of ${ }^{99 \mathrm{~m}} \mathrm{Tc}$-methoxyisobutylisonitrile in selected patient populations, which may be more difficult to image with SPECT, such as those who are obese (body mass index $\geq 30$ ), women, and those with multivessel coronary artery disease. Thus, although the initial phase 3 study is encouraging, one would hope that in the future a physiologic investigation would be embarked on in which quantitative measurements of absolute myocardial blood flow with ${ }^{18} \mathrm{~F}$ flurpiridaz were used to assess coronary stenosis severity. Clinical outcomes based on those assessments would be determined to test the hypothesis that the PET quantitative myocardial blood flow approach is superior to that of anatomic ICA (or coronary computed tomographic angiography)-based decision making regarding coronary revascularization outcomes (15).

\section{DISCLOSURE}

No potential conflict of interest relevant to this article was reported.

\section{REFERENCES}

1. Slomka PJ, Patton JA, Berman DS, Germano G. Advances in technical aspects of myocardial perfusion SPECT imaging. J Nucl Cardiol. 2009;16:255-276.

2. Depuey EG. New software methods to cope with reduced counting statistics: shorter SPECT acquisitions and many more possibilities. J Nucl Cardiol. 2009; 16:335-338.

3. Johnson NP, Sdringola S, Gould KL. Partial volume correction incorporating Rb-82 positron range for quantitative myocardial perfusion PET based on systolic-diastolic activity ratios and phantom measurements. J Nucl Cardiol. 2011;18:247-258.

4. Gewirtz H, Tawakol A, Bacharach SL. Heterogeneity of myocardial blood flow and metabolism: review of physiologic principles and implications for radionuclide imaging of the heart. J Nucl Cardiol. 2002;9:534-541.

5. Huang SC, Hoffman EJ, Phelps ME, Kuhl DE. Quantitation in positron emission computed tomography: 2 -effects of inaccurate attenuation correction. J Comput Assist Tomogr. 1979;3:804-814.

6. Hoffman EJ, Huang SC, Phelps ME. Quantitation in positron emission computed tomography: 1-effect of object size. J Comput Assist Tomogr. 1979;3:299-308.

7. Gewirtz H, Grotte GJ, Strauss HW, et al. The influence of left ventricular volume and wall motion in myocardial images. Circulation. 1979;59:1172-1177.

8. Sinusas AJ, Shi Q, Vitols PJ, et al. Impact of regional ventricular function, geometry, and dobutamine stress on quantitative ${ }^{99 \mathrm{~m}} \mathrm{Tc}$-sestamibi defect size. Circulation. 1993;88:2224-2234.

9. Kitkungvan D, Vejpongsa P, Korrane KP, Sdringola S, Gould KL. Clinical utility of enhanced relative activity recovery on systolic myocardial perfusion SPECT: lessons from PET. J Nucl Med. 2015;56:1882-1888.

10. Al Moudi M, Sun Z, Lenzo N. Diagnostic value of SPECT, PET and PET/CT in the diagnosis of coronary artery disease: a systematic review. Biomed Imaging Interv J. 2011;7:e9.

11. Pijls NHJ, Van Son JAM, Kirkeeide RK, DeBruyne B, Gould KL. Experimental basis of determining maximum coronary, myocardial, and collateral blood flow by pressure measurements for assessing functional stenosis severity before and after percutaneous transluminal angioplasty. Circulation. 1993;87:1354-1367.

12. Gould KL, Schelbert HR, Phelps ME, Hoffman EJ. Noninvasive assessment of coronary stenoses with myocardial perfusion imaging during pharmacologic coronary vasodilatation: V-detection of 47 percent diameter coronary stenosis with intravenous nitrogen-13 ammonia and emission-computed tomography in intact dogs. Am J Cardiol. 1979;43:200-208.

13. Nekolla SG, Reder S, Saraste A, et al. Evaluation of the novel myocardial perfusion positron-emission tomography tracer ${ }^{18} \mathrm{~F}-\mathrm{BMS}-747158-02$ : comparison to ${ }^{13} \mathrm{~N}$-ammonia and validation with microspheres in a pig model. Circulation. 2009; 119:2333-2342

14. Berman DS, Maddahi J, Tamarappoo BK, et al. Phase II safety and clinical comparison with single-photon emission computed tomography myocardial perfusion imaging for detection of coronary artery disease: flurpiridaz $\mathrm{F} 18$ positron emission tomography. J Am Coll Cardiol. 2013;61:469-477.

15. Packard RR, Huang SC, Dahlbom M, Czernin J, Maddahi J. Absolute quantitation of myocardial blood flow in human subjects with or without myocardial ischemia using dynamic flurpiridaz F 18 PET. J Nucl Med. 2014;55:1438-1444.

16. Gould KL, Johnson NP, Bateman TM, et al. Anatomic versus physiologic assessment of coronary artery disease: role of coronary flow reserve, fractional flow reserve, and positron emission tomography imaging in revascularization decision-making. J Am Coll Cardiol. 2013;62:1639-1653.

17. Maddahi J. Flurpiridaz F 18 MPI: recent clinical trial data. Eur Heart J Cardiovasc Imaging. 2015;16(suppl 1). 